\title{
The Susceptibility Sequence to Rheumatoid Arthritis Is a Cross-reactive B Cell Epitope Shared by the Escherichia coli Heat Shock Protein dnaJ and the Histocompatibility Leukocyte Antigen DRB10401 Molecule
}

\author{
Salvatore Albani, Julia E. Tuckwell, Lucia Esparza, Dennis A. Carson, and Jean Roudier* \\ Department of Medicine and the Sam and Rose Stein Institute for Research on Aging, University of California, San Diego, La Jolla, \\ California 92093-0945; and *Université d'Aix Marseille II. Faculté de Médecine. Hôpital de la Conception, \\ 13385 Marseille Cedex 5, France
}

\begin{abstract}
Immunological responses to bacterial heat shock proteins have been implicated in the pathogenesis of arthritis in animals and humans. The predicted amino acid sequence of dnaJ, a heat shock protein from Escherichia coli, contains an 11-amino acid segment that is homologous to the third hypervariable region of the human histocompatibility antigen (HLA) DRB10401 (formerly known as HLA Dw4), the part of the molecule that carries susceptibility to rheumatoid arthritis. To test the biological significance of this finding, we expressed and purified recombinant dnaJ ('dnaJ), and determined its immunologic cross-reactivity with HLA DRB10401. A rabbit antipeptide antiserum raised against the sequence of the third hypervariable region of HLA DRB10401 specifically bound to 'dnaJ, thus confirming that a similar sequence is expressed on the bacterial protein. Of greater consequence, an antiserum to the ${ }^{\mathrm{r}} \mathrm{dnaJ}$ protein recognized not only a peptide from the third hypervariable region of HLA DRB10401, but also the intact HLA DRB10401 polypeptide. Furthermore, the antibody to ${ }^{\mathrm{d}} \mathrm{dnaJ}$ reacted with HLA DRB10401 homozygous B lymphoblasts, but not with HLA DRB11501, DRB10101, DRB10301, and DRB10701 (formerly known as HLA Dw2, DR 1, DR 3, and DR 7, in the same order) homozygous cells. These results demonstrate that exposure to a bacterial heat shock protein can elicit antibodies against the rheumatoid arthritis susceptibility sequence in the third hypervariable region of HLA DRB10401. (J. Clin. Invest. 1992. 89:327-331.) Key words: autoimmune disease • genetic background • infection • molecular mimicry • sequence homology
\end{abstract}

\section{Introduction}

Susceptibility to rheumatoid arthritis, a chronic inflammatory joint disease, is associated with human histocompatibility antigens (HLA) DRB10401 and DRB10101 (formerly known as

Address reprint requests to Dr. Albani, Department of Medicine, S-045, University of California, San Diego, 9500 Gilman Drive, La Jolla, CA 92093-0945.

Received for publication 1 August 1991 and in revised form 30 September 1991 .

J. Clin. Invest.

(c) The American Society for Clinical Investigation, Inc.

0021-9738/92/01/0327/05 \$2.00

Volume 89, January 1992, 327-331
HLA DR 1) $(1,2)$. The molecular basis for this association is believed to be the presence of a shared amino acid sequence in the third hypervariable region of the $\beta_{1}$ chain of the disease associated alleles $(3,4)$.

How the third hypervariable regions of HLA DRB10401 and HLA DRB10101 may influence susceptibility to rheumatoid arthritis is still unknown. The binding hypothesis states that this area controls binding of an arthritogenic peptide (5). The $T$ cell interaction hypothesis suggests that direct contact between this region (or a peptide derived from this region) and the antigen receptor of a $\mathrm{T}$ cell is involved in triggering disease by expanding or deleting a particular $T$ cell population (6). These two hypotheses are not mutually exclusive.

Pathogens that are suspected to trigger HLA-associated autoimmune diseases may express proteins that share sequence identities with the third hypervariable regions of the disease associated alleles. This was first described for a Klebsiella pneumoniae nitrogenase that shares a five-amino acid identity with the third hypervariable region of the $\alpha_{1}$ chain of HLA B27 (7). We observed that the Epstein-Barr virus glycoprotein gp110 shares a six-amino acid stretch with the third hypervariable region of HLA DRB10401 and that this common stretch constitutes a T cell epitope in humans $(6,8)$.

Recent experiments have emphasized the potential role of bacterial heat shock proteins (Hsp) in the pathogenesis of arthritis in animal models and in people $(9,10)$. Heat shock proteins are major bacterial antigens. Antibodies and $T$ cells reactive with the Hsp 65 class of heat shock proteins are abundant in synovial fluids of rheumatoid arthritis patients (9).

In the present report, we show that dnaJ, a recently described heat shock protein from Escherichia coli, shares an 11-amino acid stretch of homology with the third hypervariable region of HLA DRB10401. Immunization of rabbits with recombinant dnaJ protein ( $\mathrm{r} \mathrm{dnaJ}$ ) induced the production of antibodies that react specifically with HLA DRB10401 positive $\mathrm{B}$ cells.

\section{Methods}

Subcloning, expression, and purification of ${ }^{r} d n a J$. A 1.1-kb DNA fragment containing the $E$. coli dnaJ gene was amplified by polymerase chain reaction from a recombinant phage encompassing the dnaK and dnaJ genes of $E$. coli (a kind gift of C. Georgopoulos, University of Utah) (11). The polymerase chain reaction product was subcloned in the Xba 1 site of the vector PCG808fX (New England Biolabs, Beverly, MA) and expressed as a maltose binding fusion protein that was purified by filtration on an amylose column and elution in an amylose 


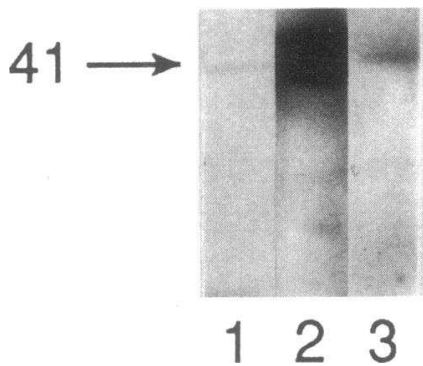

Figure 1. Expression of dnaJ from $E$. coli. Lane 1: $50 \mu \mathrm{g}$ of rdnaJ was electrophoresed on an SDS-polyacrylamide gel. After Coomassie Blue staining, a unique $41-\mathrm{kD}$ band was observed. Lanes 2 and 3: 50- $\mu \mathrm{g}$ aliquots of $\mathrm{r} d \mathrm{naJ}$ were run on an SDS gel, transferred to a nitrocellulose filter, and incubated with the same dilutions of rabbit anti-HLA DRB1040 peptide antibody alone (lane 2) or in the presence of $100 \mu \mathrm{g} / \mathrm{ml}$ of HLA DRB10401 peptide (lane 3). Binding was detected with ${ }^{125} \mathrm{I}-\mathrm{la}-$ beled protein A. Molecular mass markers are shown in kilodaltons.

buffer (12). We were unable to cut the ${ }^{\mathrm{r}} \mathrm{dnaJ}$ protein from the upstream maltose binding protein by using the cleavage site for coagulation factor $\mathrm{X}$ located at the end of the maltose binding protein. Therefore, we raised a rabbit antiserum to the fusion protein and used it to purify 'dnaJ expressed by cloning in the Xba 1 site of a pUC 19 vector. Rabbit IgG were purified on immobilized protein A (Pierce Chemical Co., Rockford, IL) and coupled to an agarose support matrix (Affi-Gel Hz, Bio-Rad Laboratories, Richmond, CA). 'dnaJ was then obtained by running the raw cellular extracts through the affinity column and eluting the bound protein in glycine buffer, $\mathrm{pH} 2.5$. Polyacrylamide gel electrophoresis of the purified product showed one unique band with the expected molecular mass of $41 \mathrm{kD}$ (Fig. 1, lane 1).

Western blotting. $100 \mu \mathrm{g}$ of purified $\mathrm{r} \mathrm{dnaJ}$, or of membrane proteins from either HLA DRB10401 or HLA DRB11501 homozygous lymphoblastoid cells, obtained by the method of Bordier (13), were loaded on a $10 \%$ SDS-polyacrylamide gel. After electrophoresis, the proteins were transferred to nitrocellulose sheets. The filters were preincubated for $1 \mathrm{~h}$ in borate-buffered saline (BBS) $\left.{ }^{1}, \mathrm{pH} 8.0\right) / 3 \%$ powdered milk; this was followed by overnight incubation at $4^{\circ} \mathrm{C}$ with the sera diluted to $1: 10$. For inhibition experiments, $100 \mu \mathrm{g} / \mathrm{ml}$ of peptide or protein was added to the antibody solution. After washing with BBS, filters were incubated with $1 \mu \mathrm{C} / \mathrm{ml}^{125} \mathrm{I}$-labeled protein A (Boehringer-Mannheim Biochemicals, Indianapolis, IN) diluted to 1:1,000, then washed with BBS, dried, and exposed to XAR film (Eastman Kodak Co., Rochester, NY) overnight at $-70^{\circ} \mathrm{C}$ using an intensifier screen.

Enzyme-linked immunoabsorbent assay (ELISA). Antibody re-

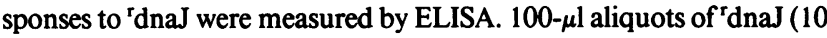
$\mu \mathrm{g} / \mathrm{ml}$ ) membrane proteins from HLA DRB10401 or DRB11501 homozygous cells $(100 \mu \mathrm{g} / \mathrm{ml})$ were used to coat ELISA plates at $4^{\circ} \mathrm{C}$ overnight. Then different dilutions of rabbit sera were added for a 2-h incubation at room temperature. After washing with BBS $/ 0.2 \%$ Tween-20, bound antibody was detected by using alkaline phosphatase-conjugated goat anti-rabbit IgG (Boehringer-Mannheim Biochemicals) diluted 1:1000. The results are expressed as the mean OD at $405 \mathrm{~nm}$ of duplicate wells. For inhibition experiments, the inhibitors $(100 \mu \mathrm{g} / \mathrm{ml})$ were incubated overnight with the serum before performance of the ELISA.

Peptides and antibodies. The peptides used in this study were synthesized by the solid-phase method of Merrifield, modified slightly as described (14), and then purified by reverse-phase HPLC on a $\mathrm{C}_{18}$ column. The sequences of the peptides are as follows: HLA DRB10401 peptide, KDLLEQKRAAVDTYC; HLA DRB 10402 (formerly known as HLA Dw10) peptide, KDILEDRAAVDTYC. When used to raise antisera, the peptides were conjugated to keyhole limpet hemocyanin by using $\mathrm{m}$-maleimidobenzoyl- $N$-hydroxyssucinamide ester (15). New Zealand white rabbits received three subcutaneous injections of $1 \mathrm{mg}$ of conjugate emulsified in complete Freund's adjuvant, with 2-wk in-

\footnotetext{
1. Abbreviation used in this paper: BBS, borate-buffered saline.
}

tervals. The rabbits were bled $4 \mathrm{~d}$ after the last injection and sera were stored at $-20^{\circ} \mathrm{C}$.

Flow cytometric analyses. Either preimmune rabbit serum, or immune serum to ${ }^{\mathrm{T}} \mathrm{dnaJ}$ were fractionated on a protein $\mathrm{A}$ column, and the bound IgG was eluted and digested with pepsin $1 \%(\mathrm{wt} / \mathrm{vol})$ at $\mathrm{pH} 3.5$ overnight. After neutralization, the residual IgG and Fc fragments were removed by a second passage over the protein A column.

Both HLA DRB10401 or DRB11501 homozygous lymphoblastoid cells were obtained from the American Type Culture Collection, Rockville, MD, and grown as described earlier (6). Lymphoblastoid cells expressing HLA DRB10101, DRB10301, and DRB10701 were kindly provided by Dr. F. Chisari, Scripps Clinic, La Jolla, CA. The cells were suspended at a density of $1 \times 10^{6} / \mathrm{ml}$ in isotonic phosphate-buffered saline (PBS) pH 7.5 containing $1 \%$ fetal bovine serum, and were incubated at $4^{\circ} \mathrm{C}$ for $30 \mathrm{~min}$ with $\left(\mathrm{Fab}^{\prime}\right)_{2}$ antibody to ${ }^{\mathrm{r}} \mathrm{dnaJ}$ or with preimmune $\left(\mathrm{Fab}^{\prime}\right)_{2}$, at concentrations ranging from 1 to $100 \mu \mathrm{g} / \mathrm{ml}$. After washing in PBS and incubation for $30 \mathrm{~min}$ at $4^{\circ} \mathrm{C}$ with fluorescein isothiocyanate (FITC) conjugated goat anti-rabbit IgG (1:160, Sigma Chemical Co., St. Louis, MO), the cells were washed and resuspended in cold PBS. Immunofluorescent cells were measured with a FACScan, using software supplied by the manufacturer (Becton, Dickinson \& Co., San Jose, CA).

\section{Results}

The QKRAA sequence from the third hypervariable region of HLA DRB10401 is contained in the predicted amino acid sequence of dnaJ, a heat shock protein from $E$. coli. The rheumatoid arthritis susceptibility sequence QKRAA is present on the predicted amino acid sequence of dnaJ, a recently described heat shock protein from $E$. coli (11). The segment of dnaJ spanning residues $61-65$ is identical to the segment of HLA DRB10401 encompassing residues 70-74. Furthermore, the following six amino acids on dnaJ, from positions 66 to 71 , are similar to the sequence on HLA DRB 10401 from positions 75 to 80 , with two identities and three conservative changes between the two proteins (Table I). These sequence data suggested that immunological cross-reactivity might exist between HLA DRB10401 and E. coli dnaJ. Subsequently studies were designed to test this prediction.

Rabbit antibody to a synthetic peptide from the third hypervariable region of HLA DRB10401 recognizes 'dnaJ. A rabbit antibody (serum 84), which was raised against a peptide from the third hypervariable region of the HLA DRB10401 chain (KDLLEQKRAAVDTYC), has been shown to be specific for the QKRAA sequence (8). This antibody (but not serum 88, specific for the HLA DRB10402 peptide (KDILEDERAAVDTYC) recognizes dnaJ, as shown by ELISA using ${ }^{\mathrm{r}} \mathrm{dnaJ}$ coated plates (Fig. 2, upper panel) and by Western blotting (Fig. 1, lane 2). Recognition is inhibited by preincubation of serum 84 with HLA DRB10401 peptide (Fig. 1, lane 3, and Fig. 2, upper

Table I. Aligned Amino Acid Sequences of the Third Hypervariable Regions of HLA DRB10401 and E. coli dnaJ in Their Areas of Homology

70 80

HLA DRB10401 KDLLE QKRAA $V$ DTY CR HNY

E. coli dnaJ VLTDS QKRAAY DQY GH $A A F$

61 71

Bold face type, identities; lightface type, conservative changes; italic type, nonconservative changes. 

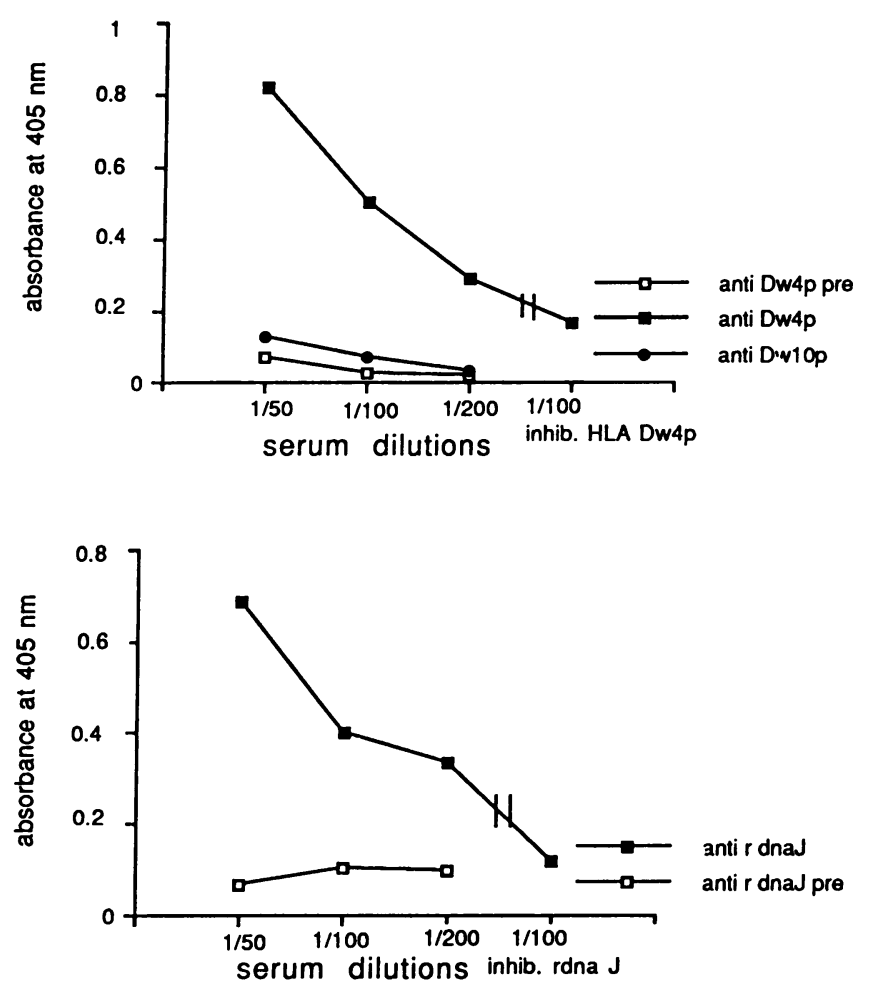

Figure 2. Cross-reactivity between dnaJ and HLA DRB10401. Upper panel: Rabbit antiserum raised against HLA DRB10401 peptide (KDLLEQKRAAVDTYC) (anti $D w 4 p$ ), recognizes 'dnaJ by ELISA, whereas preimmune serum (anti $D w 4 p$ pre) and antiserum raised against HLA DRB10402 peptide (KDILEDERAAVDTYC) (anti $D w 10 p$ ) do not. Bottom panel: Rabbit antiserum raised against ' $\mathrm{dnaJ}$ (anti dnaJ) recognizes HLA DRB10401 peptide (KDLLEQKRAAVDTYC) by ELISA, whereas preimmune serum (anti dnaJ pre) does not.

panel). These results show that the QKRAA sequence is expressed on the dnaJ protein.

Rabbit antibody to 'dnaJ binds specifically to the HLA DRB10401 chain. To evaluate whether the amino acid stretch that is shared by HLA DRB10401 and E. coli dnaJ is a B cell epitope on the intact dnaJ protein, we immunized a rabbit with rdnaJ. The resulting anti-rdnaJ serum recognized the HLA DRB10401 peptide in an ELISA (Fig. 2, lower). Furthermore, the antiserum to $\mathrm{r} d \mathrm{naJ}$ bound to membrane protein extracts from HLA DRB10401 homozygous cells as tested by ELISA (Fig. 3). Binding was inhibited by preincubation of serum with the recombinant protein $(100 \mu \mathrm{g} / \mathrm{ml})$ (Fig. 3). To confirm that the antibody to $\mathrm{r}$ naJ bound to the DRB1 chain of HLA 0401, Western blotting was performed using membrane protein extracts from HLA DRB10401 and HLA DRB11501 homozygous cells. The $\mathrm{r} d n a J$ antiserum bound a $29-\mathrm{kD}$ protein present in membrane protein extracts from HLA DRB10401 homozygous lymphoblastoid cells that was absent from HLA DRB1 1501 homozygous lymphoblasts. In some experiments, a faint $60-\mathrm{kD}$ band was also observed. Preimmune serum from the same rabbits did not bind detectably to any membrane protein. Preincubation of the antiserum with the ${ }^{r} \mathrm{dnaJ}$ protein $(100 \mu \mathrm{g} / \mathrm{ml})$ abolished reactivity with DRB10401 membranes (Fig. 4). Rabbit serum 84, that is specific for HLA DRB10401 peptide and the HLA DRB10401 chain (8), gave the same pat-

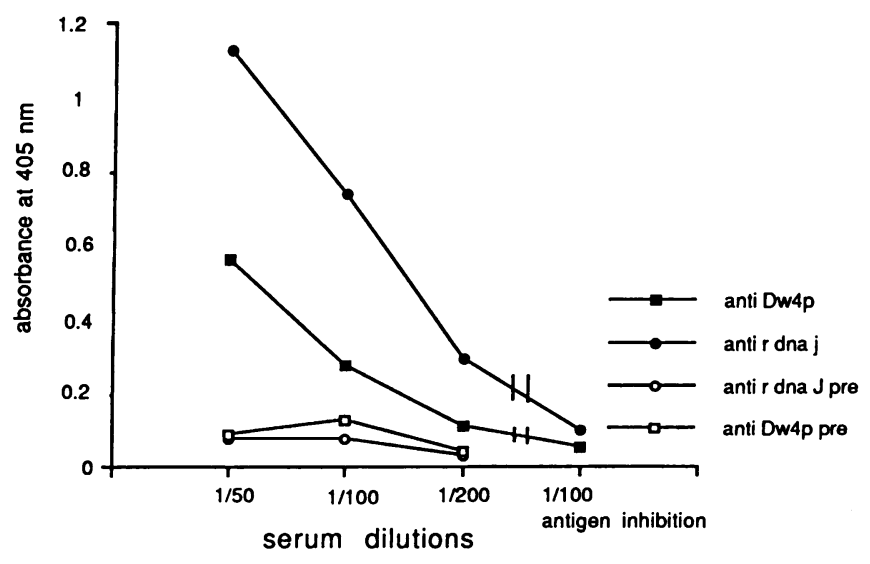

Figure 3. Binding of antibody to dnaJ to HLA DRB10401 homozygous cell membrane extracts. Antiserum raised against 'dnaJ (anti dnaJ) and antibody to HLA DRB10401 peptide

(KDLLEQKRAAVDTYC) (anti Dw4p) bind to a membrane protein extract from HLA DRB10401 homozygous lymphoblastoid cells. Preincubation of anti-dnaJ diluted 1:100 with ${ }^{\mathrm{r}} \mathrm{dnaJ}(100 \mu \mathrm{g} / \mathrm{ml})$ and preincubation of anti-Dw4p (1:100) with HLA DRB10401 peptide $(100 \mu \mathrm{g} / \mathrm{ml})$ abolish recognition (antigen inhibition). Both preimmune sera (anti-dnaJ pre, anti $D w 4 p$ pre) gave negative results.

tern, confirming that the antibody to ${ }^{\mathrm{r}} \mathrm{dnaJ}$ serum binds to HLA DRB10401 (Fig. 4).

Rabbit antibody to rdnaJ binds specifically to an $H L A$ DRB10401 homozygous cell line. It was important to determine if the B cell epitope recognized by the antibody to ${ }^{r} \mathrm{dnaJ}$ was exposed on the surface of HLA DRB10401-positive cells. To address this issue, we performed immunofluorescent analyses of viable HLA DRB10401, HLA DRB11501, HLA DRB10101, HLA DRB10301, and HLA DRB10701 homozygous $B$ lymphoblasts. To avoid any possible nonspecific binding via the Fc portion of the antibodies, the IgG fraction of the antiserum to ${ }^{\mathrm{r}} \mathrm{dnaJ}$ was digested with pepsin and the $\left(\mathrm{Fab}^{\prime}\right)_{2}$ fragments were isolated. The anti- ${ }^{r} \mathrm{dnaJ}$ antibody recognized the HLA DRB10401 protein in its native conformation on the cell surface (Table II, Fig. 5), since it bound to DRB10401 homozygous lymphoblasts, but not to DRB11501, DRB10101, DRB10301, or DRB10701 homozygous cells. The preimmune sera from the same animals did not react with HLA DRB10401 cell line (Table II).

\section{Discussion}

Susceptibility to rheumatoid arthritis is associated with the sequence QKRAA in the third hypervariable regions of the $\beta_{1}$

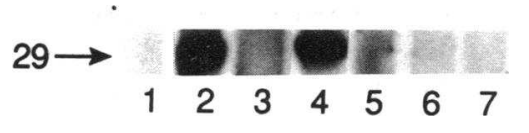

Figure 4. Specificity of the cross-reaction between dnaJ and HLA DRB10401 Lanes 1-5:

After SDS-polyacrylamide gel electrophoresis, and transfer to nitrocellulose, membrane protein extracts from HLA DRB10401 homozygous lymphoblastoid cells were incubated with anti-HLA

DRB10402 peptide (lane 1 ), anti- ${ }^{\mathrm{r}} \mathrm{dnaJ} 1 / 10$ (lane 2), anti-'dnaJ (100 $\mu \mathrm{g} / \mathrm{ml}$ ) (lane 3), anti-HLA DRB10401 peptide (lane 4), anti-HLA DRB10401 peptide 1:10 plus HLA DRB10401 peptide $100 \mu \mathrm{g} / \mathrm{ml}$ (lane 5). Lanes 6 and 7: Immunoblot of membrane protein extract from HLA DRB11501 homozygous lymphoblastoid cells, incubated with anti-rdnaJ (lane 6) and anti-HLA DRB10401 peptide (lane 7). 


\begin{tabular}{|c|c|c|c|}
\hline \multirow{2}{*}{$\begin{array}{l}\text { Lymphoblastoid } \\
\text { cell line }\end{array}$} & \multicolumn{3}{|c|}{ Antibody } \\
\hline & $\left(\mathrm{Fab}^{\prime}\right)_{2}$ anti- ${ }^{-} \mathrm{dnaJ}$ & FITC antibody only & Preimmune $\left(\mathrm{Fab}^{\prime}\right)_{2}$ \\
\hline DRB10401 & 178.02 & 30.2 & 38.04 \\
\hline DRB11501 & 34 & 27 & 57.51 \\
\hline DRB10101 & 33.7 & 13.2 & 36.7 \\
\hline DRB10301 & 58.5 & 29.5 & 77.1 \\
\hline DRB10701 & 67.3 & 23.6 & 100.4 \\
\hline
\end{tabular}

Viable HLA-DR homozygous lymphoblasts were reacted with $\left(\mathrm{Fab}^{\prime}\right)_{2}$ anti-dnaJ and developed with FITC-labeled goat anti-rabbit IgG. Controls contained the FITC-labeled goat antibody only or the preimmune $\left(\mathrm{Fab}^{\prime}\right)_{2}$. The results are expressed as the mean fluorescent intensities in arbitrary units, as measured by flow cytometry.

chain of HLA DRB10401. An identical sequence is shared by the $E$. coli heat shock protein dnaJ, while five adjacent amino acids are also homologous. As demonstrated here, antibodies against a peptide from the third hypervariable region of HLA DRB10401 and specific for the QKRAA sequence (8), also bind specifically to $\mathrm{r}$ dnaJ. In a reciprocal matter, antibodies against 'dnaJ recognize the HLA DRB10401 chain. These experiments show that HLA DRB10401 and E. coli dnaJ share an amino acid stretch that constitutes a strong cross-reactive $B$ cell epitope. This B cell epitope is exposed on the HLA DRB10401 protein in its native conformation on the cell surface, as shown by the binding of the anti- ${ }^{\mathrm{r}} \mathrm{dnaJ}$ serum to viable HLA DRB10401 lymphoblasts.

The $E$. coli dnaJ is a $40-\mathrm{kD}$ heat shock protein. The gene for dnaJ is located immediately downstream of the dnaK gene, which encodes the $E$. coli $70-\mathrm{kD}$ heat shock protein homologous to human Hsp 70 (11). E. coli dnaK and dnaJ constitute a heat shock inducible operon whose function includes protein folding and assembly (17). Of interest, human Hsp 70 maps in

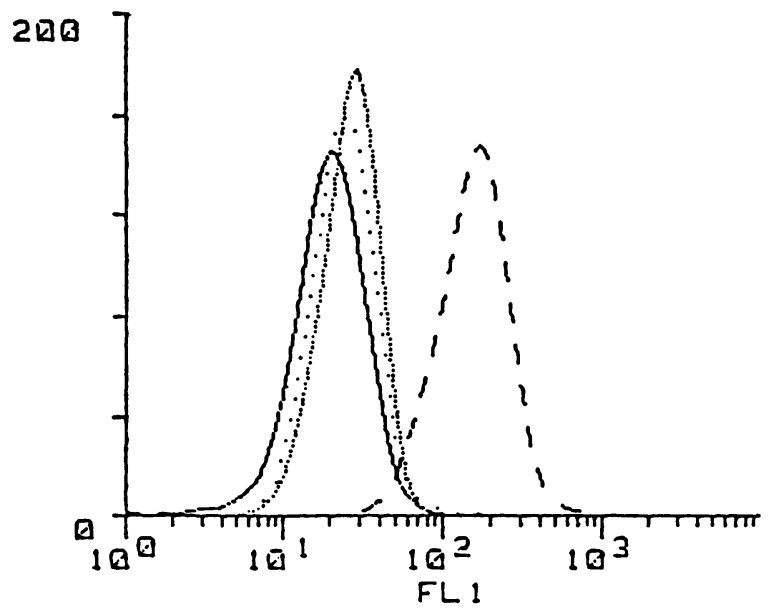

Figure 5. Binding of anti-'dnaJ antibody to HLA DRB10401 homozygous cells HLA DRB10401 homozygous B lymphoblasts were incubated in suspension with $\left(\mathrm{Fab}^{\prime}\right)_{2}$ antibody to ${ }^{\mathrm{r}} \mathrm{dnaJ}(25 \mu \mathrm{g} / \mathrm{ml})$ or with preimmune $\left(\mathrm{Fab}^{\prime}\right)_{2}$ fragments at the same concentration. Binding was detected with FITC-labeled anti-rabbit IgG, and was analyzed by flow cytometry. (- - ) HLA DRB10401 cells incubated with anti-'dnaJ $\left(\mathrm{Fab}^{\prime}\right)_{2} ;(\cdots \cdots \cdots \cdots)$ HLA DRB10401 cells incubated with preimmune serum only; (....) HLA DRB11501 cells incubated with anti-rdnaJ (Fab) ${ }_{2}$; (--)HLA DRB11501 cells incubated with preimmune serum only. the major histocompatibility complex, between the HLA DR and the HLA B region (18). It is not yet known whether there is an equivalent of $E$. coli $\mathrm{dna} J$ in the human, but the recent identification of a yeast homolog of $E$. coli dnaJ suggests that the dnaK-dnaJ operon may be present in eukaryotes (19). In this respect, it will be important to determine whether the human gene that is equivalent to dnaJ constitutes a tandem with the human Hsp 70 gene and also maps inside the HLA DRHLA B region.

The $E$. coli dnaJ would be expected to induce immune responses in humans, insofar as most people are infected with $E$. coli. In this regard, we have recently found that anti-r dnaJ antibodies are abundant in human sera (S. Albani, unpublished observations). The fact that dnaJ cross reacts with HLA DRB10401 may explain in part why antibodies to a peptide from the third hypervariable region of HLA DRB10401 have been found in the sera of patients with rheumatoid arthritis and in many normal subjects (20, J. Roudier et al., unpublished observations).

Immunologic cross reactivity between a heat shock protein and the third hypervariable region of an HLA class II molecule was originally reported by Anderson et al. (21). These investigators isolated from the peripheral blood of a patient with the tuberculoid form of leprosy, an autoreactive $T$ cell clone specific for the 65-kD heat shock protein of Mycobacterium leprae that was capable recognizing a peptide from the third hypervariable region of the $\beta_{3}$ chain of HLA DRB11501 (21). Of interest, HLA DRB11501 is associated with this particular form of leprosy. Previously, we have reported that exposure to Epstein-Barr virus infection may augment $T$ cell reactivity to the third hypervariable region of HLA DRB10401 in normal people that do not type as HLA DRB10401. In the future, it will be necessary to ascertain if systemic infection with $E$. coli induces DRB10401-reactive T cells in the human, or leads to the production of anti-DRB10401 antibodies. Preliminary results in mice suggest that immunization with ${ }^{\mathrm{r}} \mathrm{dnaJ}$ does induce $T$ cells specific for a peptide from the third hypervariable region of HLA DRB10401 (S. Albani, unpublished observation).

In summary, we have shown that the $E$. coli dnaJ, a major heat shock protein that functions during protein folding and assembly, shares sequence homology with the third hypervariable region of the HLA DRB10401 molecule. This area of HLA DRB 10401 constitutes the "shared epitope," that carries susceptibility to rheumatoid arthritis (4). Immunization with recombinant dnaJ induces antibodies that react specifically with HLA DRB10401-positive cells. Indeed, immunization with ${ }^{\mathrm{r}} \mathrm{dnaJ}$ is a simple and convenient way to produce polyclo- 
nal antibodies against the shared epitope rheumatoid arthritis that are suitable for immunofluorescent analyses. Thus, the shared epitope is mimicked by at least two proteins from microorganisms that commonly affect most people, Epstein-Barr virus gp $10(8)$ and $E$. coli $\mathrm{dnaJ}$. The evolutionary reason why this same epitope has been conserved on different $\mathrm{MHC}$ alleles and on proteins from infectious agents is still unknown. Solving this question may help reveal what triggers rheumatoid arthritis in the HLA DRB10401 background.

\section{Acknowledgments}

This work was supported in part by grant AR25443 from the National Institutes of Health, by a grant from CIBA-GEIGY Corporation, by Association pour la Recherche sur la Polyarthrite Rhumatoïde, by Fondation pour la Recherche Médicale, and by Consiglio Nazionale delle Ricerche. Dr. Roudier is the recipient of an Investigator Award from the Arthritis Foundation.

\section{References}

1. Stastny, P. 1978. Association of the B cell allo antigen DRw4 with rheumatoid arthritis. N. Engl. J. Med. 298:869-871.

2. Woodrow, J. C., F. E. Nichol, and G. Zaphiropoulos. 1981. DR antigens and rheumatoid arthritis: a study of two populations. Br. Med.J. 283:1287-1291.

3. Nepom, G. T., J. A. Hansen, and B. S. Nepom. 1987. The molecular basis for HLA class II associations with rheumatoid arthritis. J. Clin. Immunol. 7:1-9.

4. Gregersen, P., J. Silver, and R. Winchester. 1987. The shared epitope hypothesis. An approach to understanding the molecular genetics of susceptibility to rheumatoid arthritis. Arthritis Rheum. 30:1205-1213.

5. Grey, H., A. Sette, and A. Lamont. 1989. Biologic significance and therapeutic implications of antigen MHC interactions. Clin. Immunol. Immunopath. 53:47-51.

6. Roudier, J., G. Rhodes, J. Petersen, J. Vaughan, and D. A. Carson. 1988. The Epstein Barr virus glycoprotein gpl 10, a molecular link between HLA DR4, HLA DR 1 and rheumatoid arthritis. Scand. J. Immunol. 27:367-371.

7. Schwimmbeck, P., D. T. Yu, and M. B. A. Oldstone. 1987. Autoantibodies to HLA B27 in the sera of HLA B27 patients with ankylosing spondylitis or Reiter's syndrome. J. Exp. Med. 166:173-181.

8. Roudier, J., J. Petersen, G. Rhodes, J. Luka, and D. A. Carson. 1989. Susceptibility to rheumatoid arthritis maps to a T cell epitope shared by the HLA Dw4 DR beta 1 chain and the Epstein Barr virus glycoprotein gp1 10. Proc. Natl. Acad. Sci. USA. 86:5104-5108.

9. Winfield, J. 1989. Stress proteins, Arthritis and Immunity. Arthritis Rheum. 32:1497-1504.

10. Schinnick, T. M., M. H. Vadkin, and J. C. Williams. 1988. The Mycobacterium tuberculosis 65 Kilodalton antigen is a heat shock protein which corresponds to common antigen and to the $E$. coli $\mathrm{GroEl}$ protein. Infect. Immun. 56:446-451.

11. Bardwell, J., K. Tilly, E. Graig, J. King, M. Zylicz, and C. Georgopoulos. 1986. The nucleotide sequence of the Escherichia coli K12 dnaJ+ gene. J. Biol. Chem. 261:1782-1785.

12. di Guan, C., P. Li, P. Riggs, and H. Inouye. 1988. Vectors that facilitate the expression and purification of foreign peptides in Escherichia coli by fusion to maltose binding proteins. Gene. 67:21-30.

13. Bordier, C. 1981. Phase separation of integral membrane proteins in Triton X-114 J. Biol. Chem. 256:1604-1607.

14. Houghten, R., W. Chang, and C. Li. 1980. Human beta endorphin. Synthesis and characterization of analogs iodinated and tritiated at tyrosine residues 1 and 27. Int. J. Pept. Protein Res. 16:311-315.

15. Green, N., H. Alexander, A. Olson, S. Alexander, T. Shinnick, J. Sutcliffe, and $R$. Lerner. 1982. Immunogenic structure of the influenza virus hemoagglutinin. Cell. 28:477-487.

16. Devereux, J., P. Haeberli, and O. Smithies. 1984. A comprehensive set of sequence analysis programs for the VAX. Nucleic Acids Res. 12:387-395.

17. Zylicz, M., D. Ang, L. Krzysztof, and C. Georgopoulos. 1989. Initiation of $\lambda$ DNA replication with purified host and bacteriophage encoded proteins: the role of dnaK, dnaJ and grpE heat shock proteins. EMBO (Eur. Mol. Biol. Organ.) J. 8:1601-1605.

18. Sargent, C., I. Dunham, J. Trowsdale, and R. D. Campbell. 1989. Human major histocompatibility complex contains genes for the major heat shock protein HSP 70. Proc. Natl. Acad. Sci. USA. 86:1968-1971.

19. Blumberg, H., and P. Silver. 1991. A homologue of the bacterial heat shock gene DNA J that alters protein sorting in yeast. Nature (Lond.). 349:627630 .

20. Takeuchi, F., E. Kosuge, K. Matsuta, K. Nakano, K. Tokunaga, T. Juiji, and T. Miyamoto. 1990. Antibody to a specific HLA DR $\beta 1$ sequence in Japanese patients with rheumatoid arthritis. Arthritis Rheum. 33:1867-1868.

21. Anderson, D. C., W. van Schooten, M. Barry, A. Janson, T. Buchanan, and R. P. de Vries. 1988. A Mycobacterium leprae specific human T cell epitope cross reactive with an HLA DR2 peptide. Science (Wash. DC). 242:259-261. 